\title{
Loss of Coxsackie and adenovirus receptor downregulates $\alpha$-catenin expression
}

\author{
K Stecker', A Koschel', B Wiedenmann' and M Anders*,2 \\ 'Division of Gastroenterology and Hepatology, Department of Internal Medicine, Charité Medical School, Campus Virchow, Augustenburgerplatz I, Berlin \\ 13353, Germany; ${ }^{2}$ Department of Interdisciplinary Endoscopy, University Hospital Hamburg Eppendorf, Martinistraße 52, Hamburg 20246, Germany
}

BACKGROUND: The Coxsackie and adenovirus receptor (CAR) has been shown to inhibit cancer cell proliferation, migration, and invasion. The underlying mechanisms, however, are poorly understood.

METHODS: The differential gene expression in the human colon cancer cell line DLDI on RNAi-mediated functional CAR knockdown was analysed using oligo-array technology. Expression of $\alpha$-catenin was determined by quantitative RT-PCR and western blotting. Proliferation, migration, and invasion after CAR knockdown were assessed by in vitro assays, and cell morphology in a threedimensional context was evaluated using matrigel.

RESULTS: Oligo-array technology identified $\alpha$-catenin as the strongest downregulated gene after CAR knockdown. Western blotting and quantitative RT-PCR confirmed a reduced $\alpha$-catenin expression after CAR knockdown in DLDI cells and in the rat intestinal cell line IEC-6. Functionally, both cell lines showed a marked increase in proliferation, migration, and invasion on CAR knockdown. In matrigel, both cell lines formed amorphous cell clusters in contrast to well-organised three-dimensional structures of CAR-expressing vector controls. Ectopic 're'-expression of $\alpha$-catenin in DLDI and IEC-6 CAR knockdown cells reversed these functional and morphological effects.

CONCLUSION: These data suggest that an interaction of CAR and $\alpha$-catenin mediates the impact of CAR on cell proliferation, migration, invasion, and morphology.

British Journal of Cancer (2009) I II, I574- 1579. doi: I0. I038/sj.bjc.660533 I www.bjcancer.com

Published online 22 September 2009

(c) 2009 Cancer Research UK

Keywords: Coxsackie and adenovirus receptor; $\alpha$-catenin; interaction

The Coxsackie and adenovirus receptor (CAR) was first characterised as a viral attachment site on the surface of epithelial cells and was later identified as an integral component of the tight junction complex (Coyne and Bergelson, 2005). In several human carcinomas, particularly in more advanced stages, a reduced CAR presence was found, partially associated with loss of tumour differentiation, increased infiltration, and poor prognosis (Sachs et al, 2002; Matsumoto et al, 2005; Korn et al, 2006; Buscarini et al, 2007; Okegawa et al, 2007; Anders et al, 2009). On the basis of data from studies in cell lines, it has been speculated that loss of CAR promotes the proliferation, migration, and invasion of cancer cells (Okegawa et al, 2000, 2001; Bruning and Runnebaum, 2003, 2004; Huang et al, 2005; Wang et al, 2005). These phenomena are believed to result from an impaired intercellular adhesion (Okegawa et al, 2000; Bruning and Runnebaum, 2004; Wang et al, 2005). Recently, it has been shown that CAR interacts with microtubules, and therefore contributes to the cytoskeletal equilibrium and inhibits cell migration (Fok et al, 2007). A more detailed insight into the mechanisms underlying the influence of CAR on cancer cell pathobiology, however, is lacking.

Aiming to further elucidate genes that facilitate the proposed function of CAR in cancer, we determined differential gene

*Correspondence: Dr M Anders; E-mail: m.anders@uke.uni-hamburg.de Received 15 April 2009; revised 17 August 2009; accepted 24 August 2009; published online 22 September 2009 expression after CAR knockdown in the human colon cancer cell line DLD1 and assessed the functional impact of the strongest regulated gene $\alpha$-catenin.

\section{MATERIALS AND METHODS}

\section{Cell culture and generation of transfected cell lines}

The human colon cancer cell line DLD1 and the rat small intestine cell line IEC-6 were obtained from the American Type Culture Collection (Rockville, MD, USA) and from the Deutsche Sammlung von Mikroorganismen und Zellkulturen $\mathrm{GmbH}$ (Braunschweig, Germany), respectively, and were cultured in recommended growth media. Three-dimensional cultures were set up as described previously (Anders et al, 2003b). In brief, cell lines were cultured to confluence as monolayers and subsequently embedded into a commercially prepared reconstituted basement membrane from Engelbreth-Holm-Swarm tumours (BD Biosciences, Bedford, MA, USA). Cell lines with a functional CAR knockdown using CAR-specific siRNAs were generated as described previously (Anders et al, 2009). In this study, either a CAR-specific siRNA, CCAAGUACCAAGUGAAGACdTdT, or a control siRNA, CAC AAAAGUAUCGCGCAAGdTdT, cloned into the 'pSuper' vector system (Oligoengine, Seattle, WA, USA) was transfected into cell lines using Effectene (Quiagen, Hilden, Germany). The ectopic expression of $\alpha$-catenin was accomplished by the stable transfection of a human full-length $\alpha$-catenin cDNA (a kind gift from M Ozawa, 
Kagoshima University, Kagoshima, Japan) as described previously (Matsubara and Ozawa, 2004). The differential expression of CAR and the $\alpha$-catenin protein in pooled cell populations was determined by western blotting.

\section{Oligo-array analysis}

Total RNA was isolated using Trizol (Invitrogen, Karlsruhe, Germany) and reverse transcribed with oligo-dT primers and SuperScript II (Invitrogen). cDNAs generated from $200 \mathrm{ng}$ of total RNA were analysed for differential gene expression using HG U133 2.0 plus chips (Affymetrix, Santa Clara, CA, USA) and processed using GeneSpring software (Agilent Technologies, Inc., Palo Alto, CA, USA).

\section{Western blotting}

Protein lysates were obtained as previously described (Anders et al, 2003a). Equal amounts of protein lysates were loaded onto reducing Laemmli gels, immunoblotted with specific antibodies against CAR (H-300: sc-15405; Santa Cruz Biotechnology, Santa Cruz, CA, USA), $\alpha$-catenin, ZO-1 (both Zymed Laboratories, South San Francisco, CA, USA), or $\beta$-Actin (Sigma-Aldrich, Munich, Germany), and detected using the enhanced chemiluminescence system (Amersham Pharmacia, Piscataway, NJ, USA).

\section{Quantitative mRNA determination}

Total RNA was isolated using Trizol (Invitrogen) and was reverse transcribed with oligo-dT primers and SuperScript II (Invitrogen). cDNAs generated from $200 \mathrm{ng}$ of total RNA were used for real-time RT-PCR assays on a Stratagene MX3000P cycler using $\alpha$-catenin gene-specific primers (CCATGCAGGCAACATAAACTTC and AGGGTTGTAACCTGTGTAACAAG) and the qPCR MasterMix for SYBR Green I (Eurogentec Deutschland $\mathrm{GmbH}$, Köln, Germany). Quantification was carried out by the comparative $\Delta C_{\mathrm{T}}$ method normalising $C_{\mathrm{T}}$ values to $\beta$-actin (DLD1) or $18 \mathrm{~S}$ (IEC-6). cDNAs derived from $\mathrm{CHO}$ and $\mathrm{CHO}-\mathrm{CAR}$ cells were used as negative and positive controls, respectively. All experiments were carried out in triplicate and repeated at least twice.

\section{Immunofluorescence}

Immunofluorescence staining was carried out as described previously (Anders et al, 2003a). In brief, anti-CAR (H-300: sc-15405; Santa Cruz Biotechnology) and anti- $\alpha$-catenin (Zymed Laboratories) antibodies served as primary antibodies; a Cy2conjugated anti-rabbit antibody and a Cy3-conjugated anti-mouse antibody were used as secondary antibodies (Dianova $\mathrm{GmbH}$, Hamburg, Germany). Multicolour fluorescence microscopy was carried out using a Zeiss Axiophot microscope (Carl Zeiss AG, Jena, Germany).

\section{Co-immunoprecipitation}

Co-immunoprecipitations were carried out using the Immunoprecipitation Kit Protein G (Roche Diagnostics GmbH, Mannheim, Germany) according to the manufacturer's instructions. In brief, anti-CAR (H-300: sc-15405; Santa Cruz Biotechnology) and anti$\alpha$-catenin (Zymed Laboratories) antibodies served as antibodies for the initial immunoprecipitation; IgG rabbit (Dianova $\mathrm{GmbH}$ ) was used as control. Subsequent western blotting was carried out as described above.

\section{Assessment of cell proliferation}

Cells were seeded onto six-well plates $\left(4 \times 10^{5}\right.$ cells per well for DLD1 and $2 \times 10^{5}$ cells per well for IEC- 6 ) in recommended media. After $48 \mathrm{~h}$, the cells were detached using trypsin, stained with trypan blue to exclude dead cells, and counted using a haematocytometer counting chamber (VWR International, Darmstadt, Germany). All experiments were carried out in triplicate and repeated at least twice.

\section{Determination of cell migration}

Assessment of directed cancer cell migration was carried out using an AP48 48 Well Micro Chemotaxis Chamber (Neuro Probe, Gaithersburg, MD, USA). Here, 50000 cells per well in $50 \mu \mathrm{l}$ fetal calf serum (FCS)-free media were seeded onto the upper part of the chamber, whereas its lower compartment was filled with media containing $10 \%$ FCS or serum-free media as control. After $24 \mathrm{~h}$ at $37^{\circ} \mathrm{C}$, cells that migrated through the filter were fixed and stained with crystal violet containing $10 \%$ ethanol. After gentle rinsing with phosphate-buffered saline, non-migrated cells at the upper side of the filter were scraped off. Subsequently, the crystal violet dye retained on the filters was extracted with $10 \%$ acetic acid for $5 \mathrm{~min}$ at room temperature and was colorimetrically measured at $560 \mathrm{~nm}$. All experiments were carried out in triplicate and repeated at least twice.

\section{Cell invasion into matrigel}

Cells were seeded onto the top of BioCoat Matrigel Invasion Chambers (BD Biosciences) containing $8 \mu \mathrm{m}$ pore size PET membranes covered with matrigel matrix. Medium containing $10 \%$ FCS was added to the bottom well of the chambers as a chemoattractant, whereas serum-free medium was used as a control. After $24 \mathrm{~h}$ at $37^{\circ} \mathrm{C}$ and $5 \% \mathrm{CO}_{2}$, cells that invaded the matrigel-coated membrane, located at the lower membrane surface, were fixed and stained by crystal violet containing $10 \%$ ethanol. Cells of three representative areas in each well were counted at a magnification of $\times 100$. Experiments were carried out in triplicate and repeated at least twice.

\section{Statistical analysis}

Statistical calculations were carried out using GraphPad Prism software (version 4.00; GraphPad Software, Inc., San Diego, CA, USA) using an unpaired, two-tailed $t$-test, considered significant when $P<0.05$.

\section{RESULTS}

\section{Downregulation of CAR induces loss of $\alpha$-catenin}

Gene expression profiles of DLD1 cells after siRNA-mediated CAR knockdown (DLD1 ${ }^{\text {CAR-negative }}$ ) were compared with those of DLD $1^{\text {control }}$ cells (containing the pSuper vector only), using oligoarray technology. Setting a threshold of a 1.5-fold difference between the two cell lines, 1187 genes were found upregulated and 2318 genes downregulated in the DLD1 ${ }^{\text {CAR-negative }}$ cell line. Among these genes, $\alpha$-catenin displayed the highest fold change, showing an 18.54-fold lower expression in the DLD1CAR-negative cell line. Western blotting confirmed this finding at the protein level revealing a reduced $\alpha$-catenin expression in both the DLD1 ${ }^{\text {CAR-negative }}$ and the IEC-6 ${ }^{\text {CAR-negative }}$ cell lines. Furthermore, transcriptional dependence of $\alpha$-catenin by CAR was shown using reverse transcribed (RT)-PCR assays showing a reduced mRNA expression level of $\alpha$-catenin in DLD1 ${ }^{\text {CAR-negative }}$ and IEC-6 ${ }^{\text {CAR-negative }}$ cells (Figure 1A).

\section{CAR and $\alpha$-catenin co-localise, yet do not bind directly}

Co-immunofluorescence staining for CAR and $\alpha$-catenin revealed a 'honeycomb' pattern of immunopositivity at the plasma membrane showing a co-localisation of both proteins at this site in parental- (data not shown) and vector-transfected control cell 
A

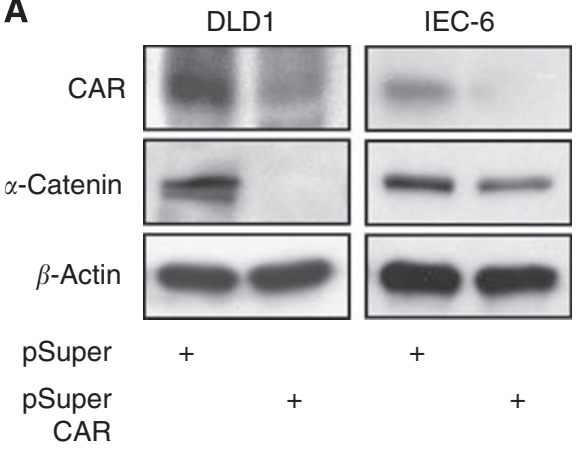

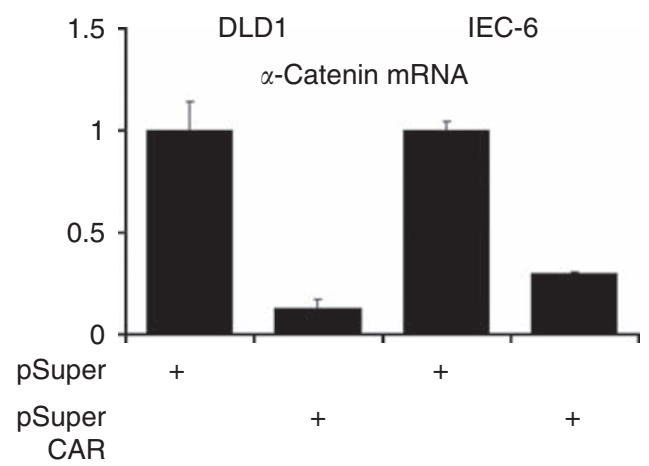

DLD1

B

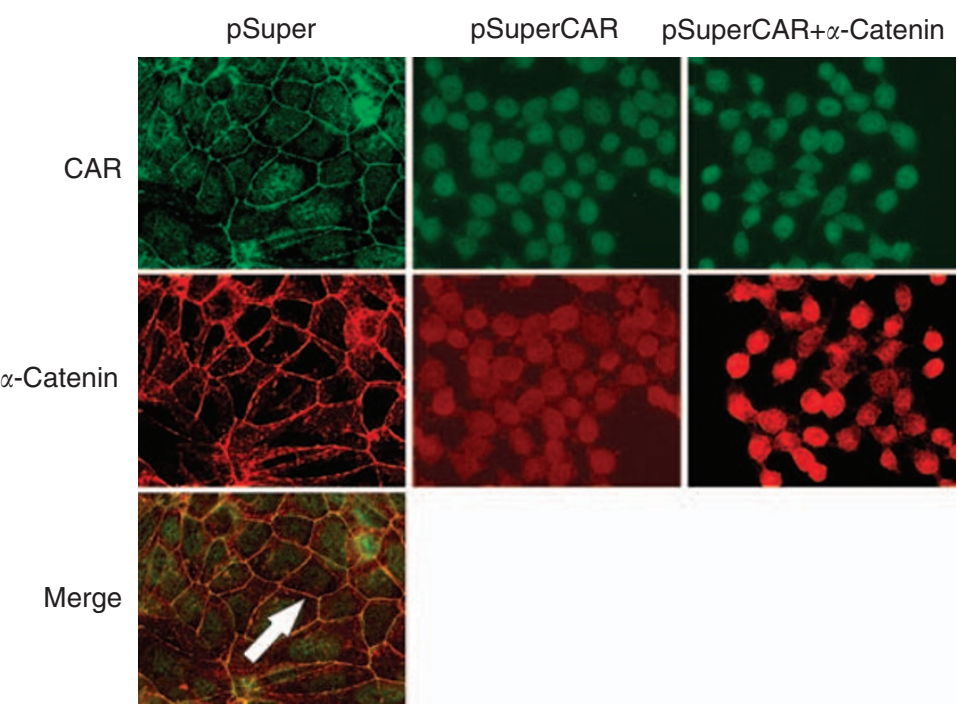

C

DLD1

IEC-6

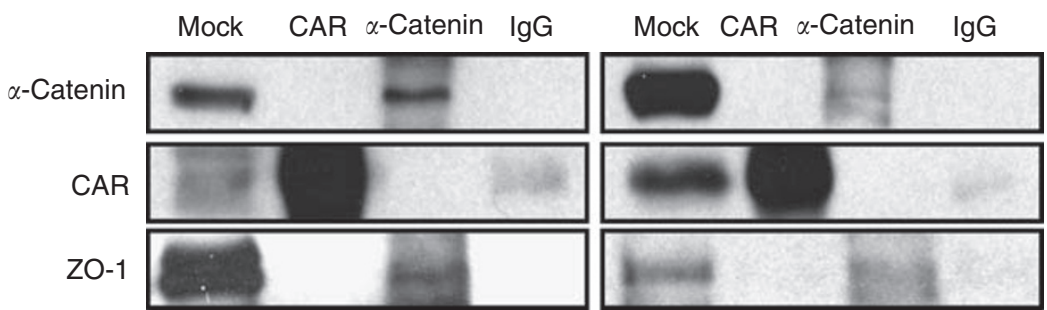

Figure I Expression and localisation of CAR and $\alpha$-catenin. Protein expression levels of CAR, $\alpha$-catenin, and $\beta$-actin were analysed by western blotting (A, left panel). Transcriptional regulation of $\alpha$-catenin after CAR downregulation shown by QRT-PCR with expression levels in mock vector-transfected controls set to I (A, right panel). Immunofluorescence co-staining for CAR and $\alpha$-catenin reveals the presence of both proteins at the plasma membrane (arrow) in mock vector-transfected controls (left panels), loss of protein presence at the plasma membrane after CAR downregulation (intracellular signal intensity equals controls that contain secondary antibodies only) (middle panels), and intracellular immunopositivity for $\alpha$-catenin after 're'-expression of $\alpha$-catenin (right panels) (magnification $\times 630)(B)$. Co-immunoprecipitations indicate no binding between CAR and $\alpha$-catenin, yet confirm an interaction of $\alpha$-catenin and ZO-I (C).

lines. In contrast, DLD1 ${ }^{\text {CAR-negative }}$ and IEC-6 ${ }^{\text {CAR-negative }}$ cells showed a weak intracellular signal only, comparable with that of controls stained by secondary antibodies only (data not shown). After the ectopic expression of $\alpha$-catenin in DLD $1^{\text {CAR-negative }}$ and IEC- 6 CAR-negative cell lines, a strong intracellular immunopositivity was noted for $\alpha$-catenin, yet no signal was detected at the plasma membrane (Figure 1B). To elucidate whether CAR and $\alpha$-catenin proteins bind to each other, we performed co-immunoprecipitations. These analyses showed binding neither when carried out with the anti-CAR antibody nor when carried out with the anti- $\alpha$ catenin antibody. To test whether our experimental conditions are sufficient to reveal inter-protein binding, additional western blotting was carried out with an anti-ZO-1 antibody, a known binding partner of $\alpha$-catenin (Imamura et al, 1999). These experiments confirmed the binding of $\mathrm{ZO}-1$ to $\alpha$-catenin in both cell lines (Figure 1C).

\section{Impact of CAR and $\alpha$-catenin on cellular morphology}

When cultured under conventional conditions, slight changes in cell shape were noted for both cell lines: DLD1 ${ }^{\text {CAR-negative }}$ cells appeared rounder and smaller compared with controls, whereas IEC- $6^{\text {CAR-negative }}$ cells showed a spindlier shape with less intercellular contact. More dramatic differences were observed 
A
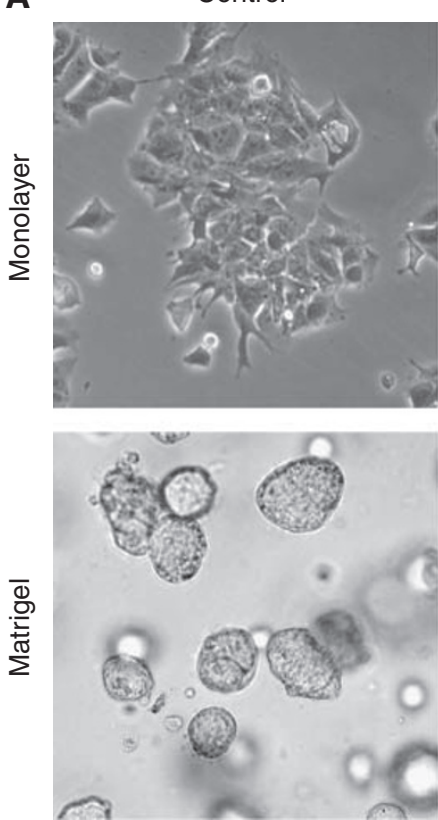

B
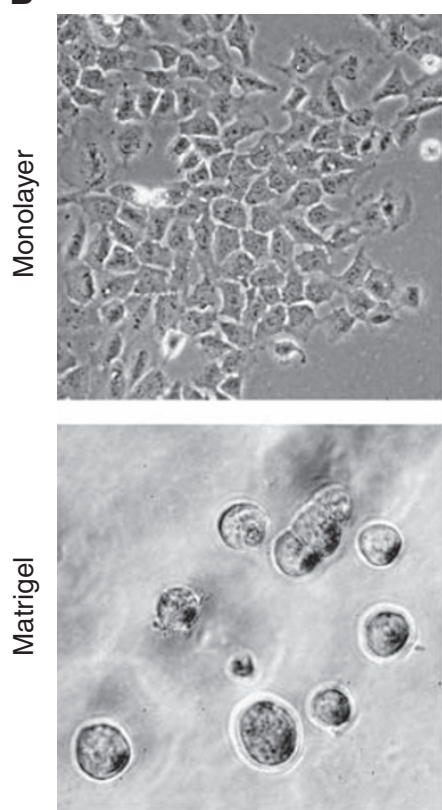

pSuperCAR
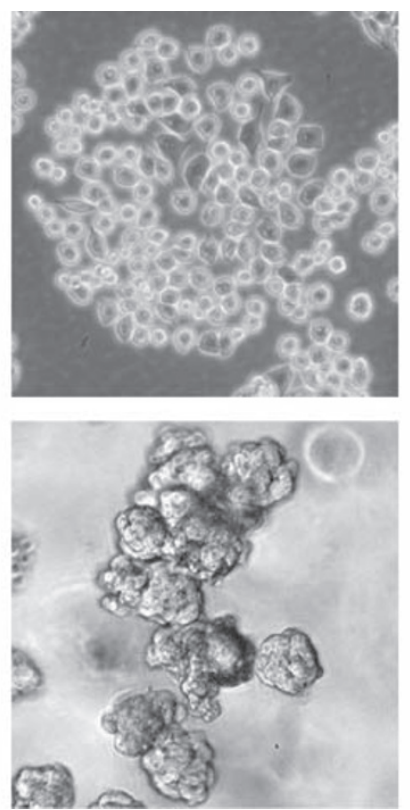

pSuperCAR
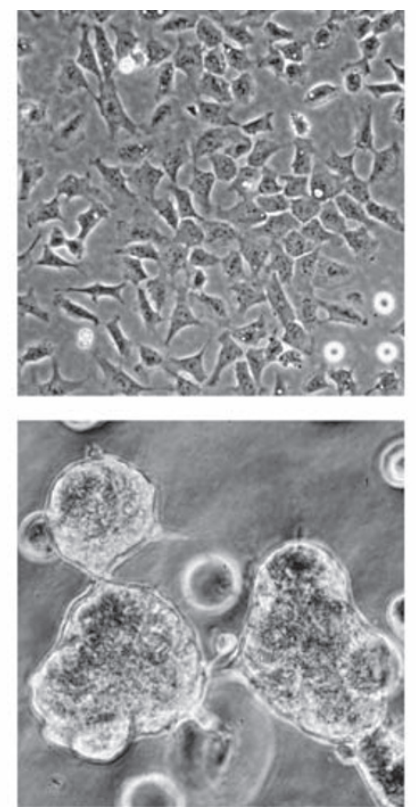

CAR - / $\alpha$-Cat. +
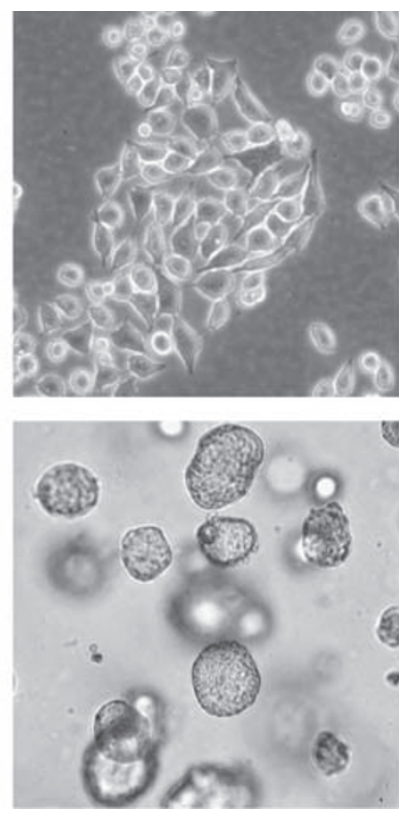

CAR -/ $\alpha$-Cat. +
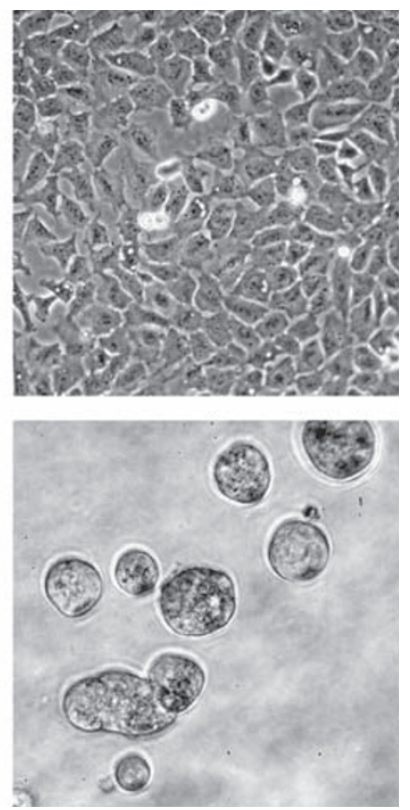

Figure 2 Impact of CAR and $\alpha$-catenin on cellular morphology. Cells after CAR knockdown appear round and smaller compared with controls (DLDI) or display less intercellular attachment sites (IEC-6). In matrigel, cells after CAR knockdown form amorphous clusters in contrast to the organised-appearing formations of matching controls. Ectopic 're'-expression of $\alpha$-catenin partially reverses the effects seen after CAR knockdown when grown as a monolayer, and particularly results in organised cell formations similar to those of vector controls when cultured in matrigel. Images show representative results for

DLDI (A) and IEC-6 (B).

when cells were cultured in matrigel, enabling cells to form threedimensional structures. Both cell lines formed amorphous clusters in sharp contrast to the well-organised-appearing formations of control cell lines. The ectopic 're'-expression of $\alpha$-catenin in DLD1 ${ }^{\text {CAR-negative }}$ and IEC- $6^{\text {CAR-negative }}$ partially reversed the effects of CAR knockdown, resulting in bigger-, flatter-, and angularappearing cells. When grown in matrigel, these cell lines established organised formations similar to those of DLD1 ${ }^{\text {control }}$ and IEC- $6^{\text {control }}$ (Figure 2).

\section{Impact of CAR on cell proliferation, migration, and invasion is mediated by $\alpha$-catenin}

To clarify the functional influence of the CAR- $\alpha$-catenin interaction, we performed in vitro assays after CAR knockdown and the ectopic 're'-expression of $\alpha$-catenin. Assessment of cell proliferation revealed significantly higher cell numbers in DLD1 1 CAR-negative

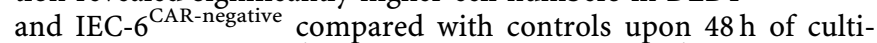
vation (DLD1 ${ }^{\text {CAR-negative }} P=0.0017$; IEC-6 CAR-negative $P=0.0129$ ). 
A

Proliferation
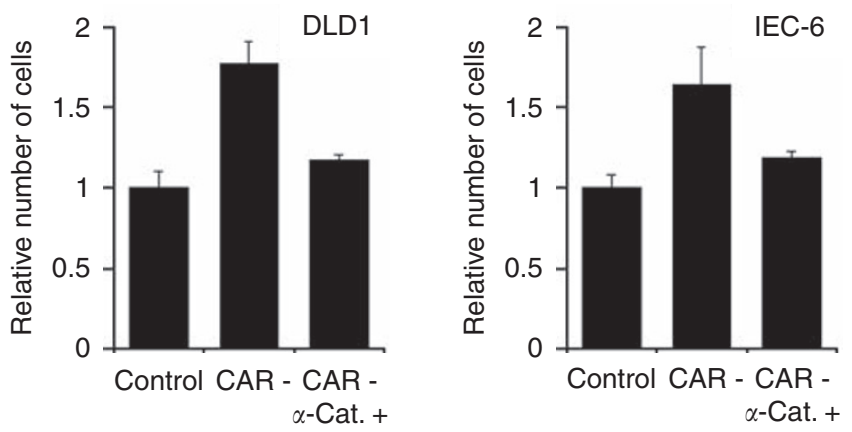

B

Migration
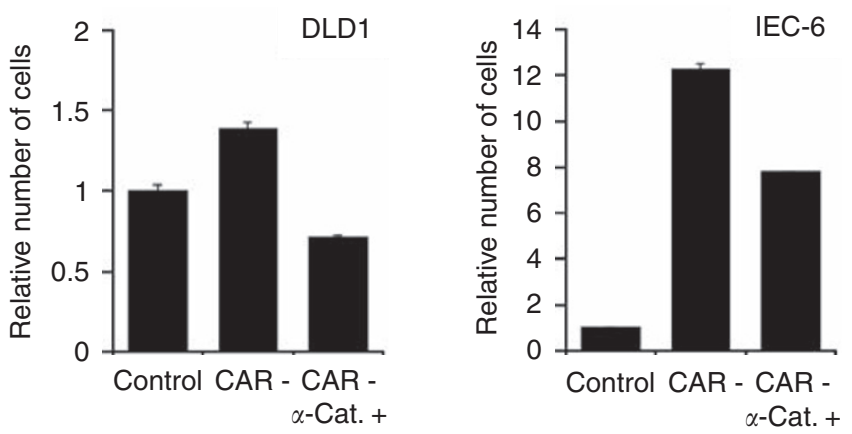

C

Invasion
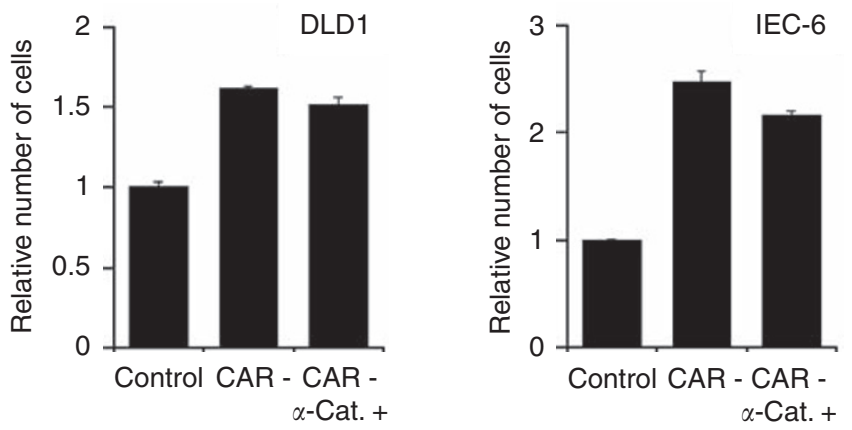

Figure 3 Functional impact of CAR downregulation and $\alpha$-catenin 're'expression in DLDI and IEC-6 cell lines. Proliferation (A), migration (B), and invasion (C) in DLDI and IEC-6 were determined after CAR knockdown and 're'-expression of $\alpha$-catenin in comparison with controls.

Ectopic 're'-expression of $\alpha$-catenin in DLD1 ${ }^{\text {CAR-negative }}$ and IEC-6 6 CAR-negative resulted in significantly lower cell numbers (DLD1 ${ }^{\text {CAR-negative/ } \alpha \text {-catenin }} \quad P=0.0022 ; \quad$ IEC- 6 CAR-negative/ $\alpha$-catenin $P=0.0319$ ) (Figure $3 \mathrm{~A}$ ). Using migration assays, DLD1 CAR-negative and IEC- 6 CAR-negative showed significantly increased migratory properties compared with vector-control cell lines (DLD1 ${ }^{\text {CAR-negative }}$ $P=0.0005$; IEC-6 $\left.{ }^{\text {CAR-negative }} P<0.0001\right)$. In contrast, ectopic 're'-expression of $\alpha$-catenin significantly decreased the number of migrating cells for DLD $1{ }^{\text {CAR-negative/ } \alpha \text {-catenin }}$ and IEC- $6^{\text {CAR-negative/ } \alpha \text {-catenin }}$ (both: $P<0.0001$ ) (Figure 3B). To test whether cells migrate in an FCS-directed manner, we included FCS-free medium controls for each of the tested cell lines, without noting cell migration in any case (data not shown). The number of cells invading into matrigel compared with vector controls increased significantly for both DLD1 ${ }^{\text {CAR-negative }}(P<0.0001)$ and IEC-6 ${ }^{\text {CAR-negative }}$ $(P<0.0001)$ cell lines. Ectopic 're'-expression of $\alpha$-catenin impaired cell invasion significantly (DLD1 1 CAR-negative/ $\alpha$-catenin $P=0.0318$; IEC-6 $\left.{ }^{\text {CAR-negative } / \alpha \text {-catenin }} P=0.0129\right)$, yet was not sufficient to reduce the number of invading cells to the level of $\mathrm{DLD1}^{\text {control }}$ and IEC-6 ${ }^{\text {control }}$ cell lines (Figure $3 \mathrm{C}$ ).

\section{DISCUSSION}

This study marks the first report of an impaired $\alpha$-catenin expression after downregulation of CAR. On the basis of our data, it may be speculated that this phenomena crucially affects the inhibitory effect of CAR on cell proliferation, migration, and invasion. Moreover, our observations reveal that loss of CAR is potentially accompanied by substantial changes in cellular morphology that may also be explained by an impaired presence of $\alpha$-catenin.

Our findings reveal that a reduced $\alpha$-catenin mRNA expression represents the most prominent feature after downregulation of CAR. This observation was further validated by our finding of reduced $\alpha$-catenin protein presence in DLD1 and IEC- 6 cell lines upon downregulation of CAR. As DLD1 represents a human cancer cell line and IEC-6 a rat non-transformed small intestinal cell line, it may be speculated that our results account for a more general phenomenon. However, differential protein expression of $\alpha$-catenin after CAR downregulation differs between cell lines: after a transient transfection of a CAR-specific siRNA, reduced $\alpha$-catenin protein expression was noted in AGS (gastric cancer) and SW480 (colon cancer), whereas in T84 and HCT116 cells, only a moderate decline was observed and no changes were noted in Caco2 (all colon cancer; data not shown). The underlying mechanisms of these differences remain to be elucidated. Transcriptional regulation of $\alpha$-catenin expression after CAR downregulation potentially marks the main regulatory measure, as shown by quantitative RT-PCR. However, whether the $\alpha$-catenin promoter becomes inactivated upon loss of CAR or changes in RNA stability occur, remains to be elucidated.

The previously proposed inhibitory effects of CAR on cancer cell growth and motility have been suggested to result from an impaired intercellular adhesion (Okegawa et al, 2000; Bruning and Runnebaum, 2004; Wang et al, 2005). Our data show that loss of CAR impairs the expression of $\alpha$-catenin, a protein that has been extensively studied for its inhibition of cancer cell growth and motility (Scott and Yap, 2006). Moreover, loss of $\alpha$-catenin is frequently described in carcinomas, for example, colon cancer, significantly correlating with depth of invasion, presence of metastasis, and an adverse clinical outcome (Ropponen et al, 1999). In line with these reports, we noted an increased proliferation, migration, and invasion after loss of CAR and $\alpha$-catenin. Furthermore, our data show that 're'-expression of $\alpha$-catenin is sufficient to markedly reverse these effects. Therefore, it may be speculated that the functional effects on cell growth and motility after loss of CAR are crucially mediated by a reduced expression of $\alpha$-catenin. However, cell invasion has been reduced to a small extent only on re-expression of $\alpha$-catenin. Given that our experimental setting sufficiently reflects cell invasion, we found that a CAR- $\alpha$-catenin interaction seems to be less important in this context compared with cell proliferation and migration.

Both $\alpha$-catenin and, recently, CAR have been shown to interact with the cytoskeleton (Scott and Yap, 2006; Fok et al, 2007). In agreement with these studies, we noted substantial changes in cell morphology, particularly when using three-dimensional culturing, of cell lines after CAR knockdown. The impact of $\alpha$-catenin on this phenomenon was shown by the 're-'expression of $\alpha$-catenin, which resulted in the formation of organised cell formations as opposed to amorphous clusters after CAR knockdown.

Co-immunofluorescence staining for CAR and $\alpha$-catenin showed a co-localisation of both proteins at the plasma membrane in parental- and vector-transfected control cell lines. However, the 're'-expression of $\alpha$-catenin in CAR-negative cell lines resulted in 
an intracellular accumulation of $\alpha$-catenin, yet no 're'-distribution to the plasma membrane. Therefore, we hypothesised that CAR may recruit $\alpha$-catenin to the plasma membrane. Yet, coimmunoprecipitation using either an anti-CAR or an anti$\alpha$-catenin antibody failed to proof a binding of these proteins. Therefore, reduced presence of other proteins that link $\alpha$-catenin to the plasma membrane may account for the intracellular accumulation of $\alpha$-catenin seen here. Nevertheless, binding of CAR to $\alpha$-catenin may not be excluded entirely, as it might be weak and/or transient and not be detectable biochemically. Herein, our findings are potentially in line with a recent study showing that $\alpha$-catenin, rather than forming a stable link to actin, acts as a regulator of actin dynamics and that its binding to the E-cadherin- $\beta$-catenin complex is transient and mutually exclusive of its interaction with F-actin (Drees et al, 2005).

\section{REFERENCES}

Anders M, Christian C, McMahon M, McCormick F, Korn WM (2003a) Inhibition of the Raf/MEK/ERK pathway up-regulates expression of the coxsackievirus and adenovirus receptor in cancer cells. Cancer Res 63: 2088-2095

Anders M, Hansen R, Ding RX, Rauen KA, Bissell MJ, Korn WM (2003b) Disruption of $3 \mathrm{D}$ tissue integrity facilitates adenovirus infection by deregulating the coxsackievirus and adenovirus receptor. Proc Natl Acad Sci USA 100: $1943-1948$

Anders M, Vieth M, Rocken C, Ebert M, Pross M, Gretschel S, Schlag PM, Wiedenmann B, Kemmner W, Hocker M (2009) Loss of the coxsackie and adenovirus receptor contributes to gastric cancer progression. Br J Cancer 100: 352-359

Bruning A, Runnebaum IB (2003) CAR is a cell-cell adhesion protein in human cancer cells and is expressionally modulated by dexamethasone, TNFalpha, and TGFbeta. Gene Therapy 10: 198-205

Bruning A, Runnebaum IB (2004) The coxsackie adenovirus receptor inhibits cancer cell migration. Exp Cell Res 298: 624-631

Buscarini M, Quek ML, Gilliam-Hegarich S, Kasahara N, Bochner B (2007) Adenoviral receptor expression of normal bladder and transitional cell carcinoma of the bladder. Urol Int 78: 160-166

Coyne CB, Bergelson JM (2005) CAR: a virus receptor within the tight junction. Adv Drug Deliv Rev 57: 869-882

Drees F, Pokutta S, Yamada S, Nelson WJ, Weis WI (2005) Alpha-catenin is a molecular switch that binds E-cadherin-beta-catenin and regulates actin-filament assembly. Cell 123: $903-915$

Fok PT, Huang KC, Holland PC, Nalbantoglu J (2007) The Coxsackie and adenovirus receptor binds microtubules and plays a role in cell migration. J Biol Chem 282: $7512-7521$

Huang KC, Altinoz M, Wosik K, Larochelle N, Koty Z, Zhu L, Holland PC, Nalbantoglu J (2005) Impact of the coxsackie and adenovirus receptor (CAR) on glioma cell growth and invasion: requirement for the C-terminal domain. Int $J$ Cancer 113: 738-745

Imamura Y, Itoh M, Maeno Y, Tsukita S, Nagafuchi A (1999) Functional domains of alpha-catenin required for the strong state of cadherin-based cell adhesion. J Cell Biol 144: $1311-1322$
In conclusion, it may be speculated that the suggested tumour suppressive function of CAR is at least in part mediated by regulating the $\alpha$-catenin expression. Underlying regulatory mechanisms remain to be elucidated.

\section{ACKNOWLEDGEMENTS}

We thank Dr Masayuki Ozawa (Department of Biochemistry, Faculty of Medicine, Kagoshima University, Kagoshima, Japan) for providing the human full-length $\alpha$-catenin cDNA and Dr Ute Ungethüm (Labor für Funktionelle Genomforschung, Charité, Berlin, Germany) for the oligo-array analysis. This study was supported by a grant from the Deutsche Forschungsgemeinschaft to MA (An 347/3-2).

Korn WM, Macal M, Christian C, Lacher MD, McMillan A, Rauen KA, Warren RS, Ferrell L (2006) Expression of the coxsackievirus- and adenovirus receptor in gastrointestinal cancer correlates with tumor differentiation. Cancer Gene Ther 13: $792-797$

Matsubara S, Ozawa M (2004) Expression of alpha-catenin in alphacatenin-deficient cells results in a reduced proliferation in threedimensional multicellular spheroids but not in two-dimensional monolayer cultures. Oncogene 23: 2694-2702

Matsumoto K, Shariat SF, Ayala GE, Rauen KA, Lerner SP (2005) Loss of coxsackie and adenovirus receptor expression is associated with features of aggressive bladder cancer. Urology 66: $441-446$

Okegawa T, Li Y, Pong RC, Bergelson JM, Zhou J, Hsieh JT (2000) The dual impact of coxsackie and adenovirus receptor expression on human prostate cancer gene therapy. Cancer Res 60: 5031-5036

Okegawa T, Pong RC, Li Y, Bergelson JM, Sagalowsky AI, Hsieh JT (2001) The mechanism of the growth-inhibitory effect of coxsackie and adenovirus receptor (CAR) on human bladder cancer: a functional analysis of car protein structure. Cancer Res 61: 6592-6600

Okegawa T, Sayne JR, Nutahara K, Pong RC, Saboorian H, Kabbani W, Higashihara E, Hsieh JT (2007) A histone deacetylase inhibitor enhances adenoviral infection of renal cancer cells. J Urol 177: $1148-1156$

Ropponen KM, Eskelinen MJ, Lipponen PK, Alhava EM, Kosma VM (1999) Reduced expression of alpha catenin is associated with poor prognosis in colorectal carcinoma. J Clin Pathol 52: 10-16

Sachs MD, Rauen KA, Ramamurthy M, Dodson JL, De Marzo AM, Putzi MJ, Schoenberg MP, Rodriguez R (2002) Integrin alpha(v) and coxsackie adenovirus receptor expression in clinical bladder cancer. Urology 60: $531-536$

Scott JA, Yap AS (2006) Cinderella no longer: alpha-catenin steps out of cadherin's shadow. J Cell Sci 119: 4599-4605

Wang B, Chen G, Li F, Zhou J, Lu Y, Ma D (2005) Inhibitory effect of coxsackie adenovirus receptor on invasion and metastasis phenotype of ovarian cancer cell line SKOV3. J Huazhong Univ Sci Technolog Med Sci 25: $85-87,93$ 